\title{
A Rare Case of Dissemination of Primary Spinal Glioblastoma
}

\author{
Deniz Sirinoglu ${ }^{1} \quad$ Buse Sarigul ${ }^{1, \odot} \quad$ Ozan Baskurt $^{1} \quad$ Mehmet Volkan Aydin $^{1}$ \\ ${ }^{1}$ Department of Neurosurgery, Prof. Dr. Cemil Tascioglu State \\ Hospital, Istanbul, Turkey \\ Address for correspondence Deniz Sirinoglu, MD, Department of \\ Neurosurgery, Prof. Dr. Cemil Tascioglu State Hospital, Istanbul, \\ Turkey (e-mail: denizsirinoglu77@hotmail.com).
}

Indian J Neurosurg 2021;10:162-164.

\begin{abstract}
Background Primary spinal glioblastoma is a rare lesion which constitutes only $1.5 \%$ of all spinal tumors. Diagnosis is challenging due to absence of any radiological hallmark of the disease. Even though surgery combined with chemoradiotherapy is the optimal management for these tumors, prognosis is still poor. Dissemination of glioblastoma is reported several times in the literature, which is correlated with worse prognosis and outcome. This case report that we are presenting is the first case where dissemination of primary spinal glioblastoma dissemination is limited only to spinal cord, without intracranial invasion, and regression was achieved with chemoradiotherapy.

Case Report In this article, a patient with primary spinal glioblastoma who presented with paraparesia is presented. Patient underwent surgery for resection of the tumor and received adjuvant radiotherapy. However, 9 months postoperatively, dissemination was detected in epidural and subdural spaces of spinal canal, with concomitant compression of spinal cord. There was no radiographically detected lesion in cranial MRI. He underwent second session of radiotherapy, combined with chemotherapy and steroid. Five months later, regression of metastatic disseminated lesions was observed.

Keywords

- spinal glioblastoma

- dissemination

- intramedullary tumor

Conclusion Primary spinal glioblastoma is a rare pathology and known to have a poor prognosis, notably with dissemination of the disease. Even though further biomolecular studies are necessary to explain the pathophysiology better, chemotherapy and radiotherapy may be effective in regression of disseminated lesions.
\end{abstract}

\section{Introduction}

Glioblastoma of the spinal cord is a very rare entity, consisting of 2 to $4 \%$ of all glial tumors of the central nervous system (CNS). ${ }^{1}$ This rarity may be explained by the lower number of neuroglial cells in the spinal cord, in contrast to brain where glioblastoma is the most common primary neoplasm. ${ }^{1}$ Most intramedullary located spinal tumors comprise gliomas, and histopathological grading is the most important prognosticator for these tumors. ${ }^{2}$

published online July 15, 2021
DOI https://doi.org/

$10.1055 / \mathrm{s}-0041-1731974$ ISSN 2277-954X
Main cause of mortality of spinal glioblastoma is reported to be intracranial metastasis and respiratory failure. On the other hand, pathophysiology of CNS dissemination and metastasis of glioblastoma is not well understood yet. Cerebrospinal fluid (CSF) dissemination and growth along nerve fiber tracts are hypothesized to cause metastatic course of the disease. ${ }^{3}$ Ventricular system exposure and iatrogenic spread of tumor into CSF during surgery for intracranial tumor resection is also postulated to be a risk factor for dissemination. ${ }^{4}$

(C) 2021. Neurological Surgeons' Society of India.

This is an open access article published by Thieme under the terms of the Creative Commons Attribution-NonDerivative-NonCommercial-License, permitting copying and reproduction so long as the original work is given appropriate credit. Contents may not be used for commercial purposes, or adapted, remixed, transformed or built upon. (https://creativecommons.org/licenses/by-nc-nd/4.0/).

Thieme Medical and Scientific Publishers Pvt. Ltd. A-12, 2nd Floor, Sector 2, Noida-201301 UP, India 
In this report, a patient with primary spinal glioblastoma, who developed leptomeningeal dissemination 9 months after surgery combined with radiotherapy, is presented. There are several reported cases either on spinal dissemination of intracranial glioblastoma or intracranial dissemination of primary spinal glioblastoma. However, to the best of our knowledge, this is the first case report that presents spinal dissemination from a primary spinal glioblastoma without intracranial invasion. Moreover, regression of leptomeningeal metastases could be achieved with chemoradiotherapy.

\section{Case Report}

A 32-year-old male patient presented with weakness in both legs, aggravated by standing and walking, together with tingling sensation below knees for 2 months. His past medical history and family history were clear. In his neurological examination, hip flexion muscle strength was 3/5, ankle dorsiflexion and hallucis longus extension muscle strength were $0 / 5$ bilaterally. There was dysesthesia under patella, predominantly in left. Patellar and Achilles tendon reflexes were hypoactive. He did not have neither urinary nor fecal incontinence.

An intradural intramedullary lesion at T1-2 vertebral level was detected via spinal MR imaging (-Fig. 1A, B). On T1 contrast-weighted sequence, lesion showed hyperintensity and homogenous contrast enhancement with well-defined borders. Perilesional edema was prominent, which was better detected on T2-weighted sequences. CT scan of spine was clear of any osseous pathology. Our initial differential diagnosis included transvers myelitis, ependymoma, and glial tumor.

Gross total excision was achieved via surgery, and histopathological examination of the lesion revealed glioblastoma with IDH-NOS pattern. It showed GFAP and p53 mutation with high cellularity and nuclear atypia. Patient underwent radiotherapy postoperatively. Physical therapy was initiated immediately after the operation, however there was no improvement in neurological outcome.

Nine months after the surgery, with a complaint of severe back pain, patient's MRI scan revealed extensive contrast enhancement in posterior compartment of subarachnoid and epidural space from lower cervical through lumbar levels which caused significant compression of spinal cord ( - Fig. 1C-H). Brain MRI did not show any additional lesion. For differential diagnosis, dissemination of glial tumor and radiation necrosis was considered. Patient underwent second dose of radiotherapy together with $150 \mathrm{mg} / \mathrm{m}^{2}$ dosing of temozolomide. He also received oral dexamethasone therapy, $16 \mathrm{mg}$ per day. Operation was not intended due to disseminated course of the pathology. Five months after adjuvant therapy, his control spinal MRI scan revealed regression of lesions, especially in cervical level (-Fig. 2). Compression over spinal cord was decreased, and patient's back pain decreased.

\section{Discussion}

Spinal dissemination of intracranial glioblastoma is shown several times in literature. ${ }^{4,5}$ On the other hand, intracranial invasion of primary spinal glioblastoma are reported less frequently. ${ }^{6,7}$ Glioblastoma is shown to metastasize in subarachnoid space and infiltrate myelin cells. ${ }^{8}$ In this case report, dissemination of primary spinal glioblastoma along spinal canal without any intracranial invasion is presented. To the best of our knowledge, this is the first case report in which dissemination is only limited to spinal cord, and even though overall outcome is poor, regression of metastatic lesions is achieved via chemotherapy and radiotherapy.
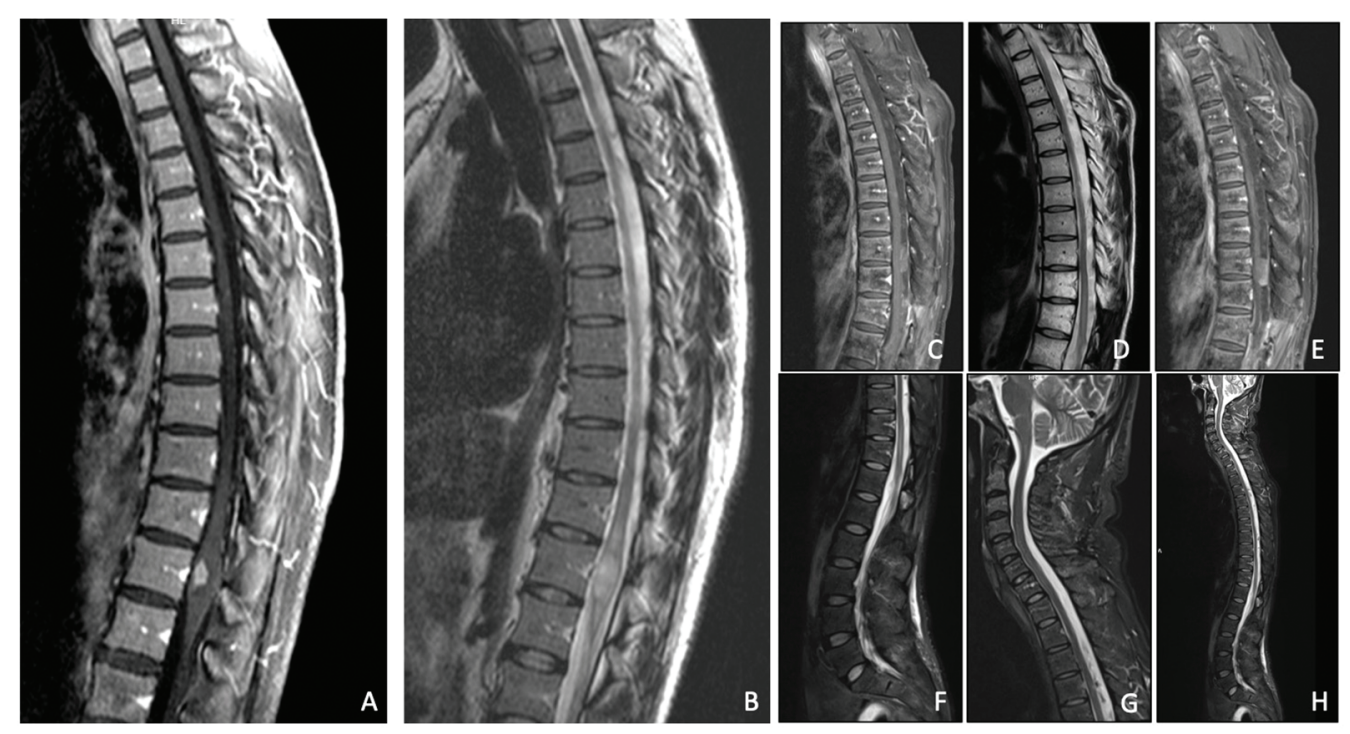

Fig. 1 (A, B). T1 contrast-weighted dorsal spinal MRI. T2-weighted sequence sagittal dorsal spinal MRI shows perilesional edema and consequent dilatation of spinal cord. (C-H). Nine months after the operation and radiotherapy, T2-weighted imaging (T2WI) of the spinal cord shows hyperintense lesions in epidural and subarachnoid space, which causes compression and narrowing in spinal cord, predominantly in cervicothoracic level. 

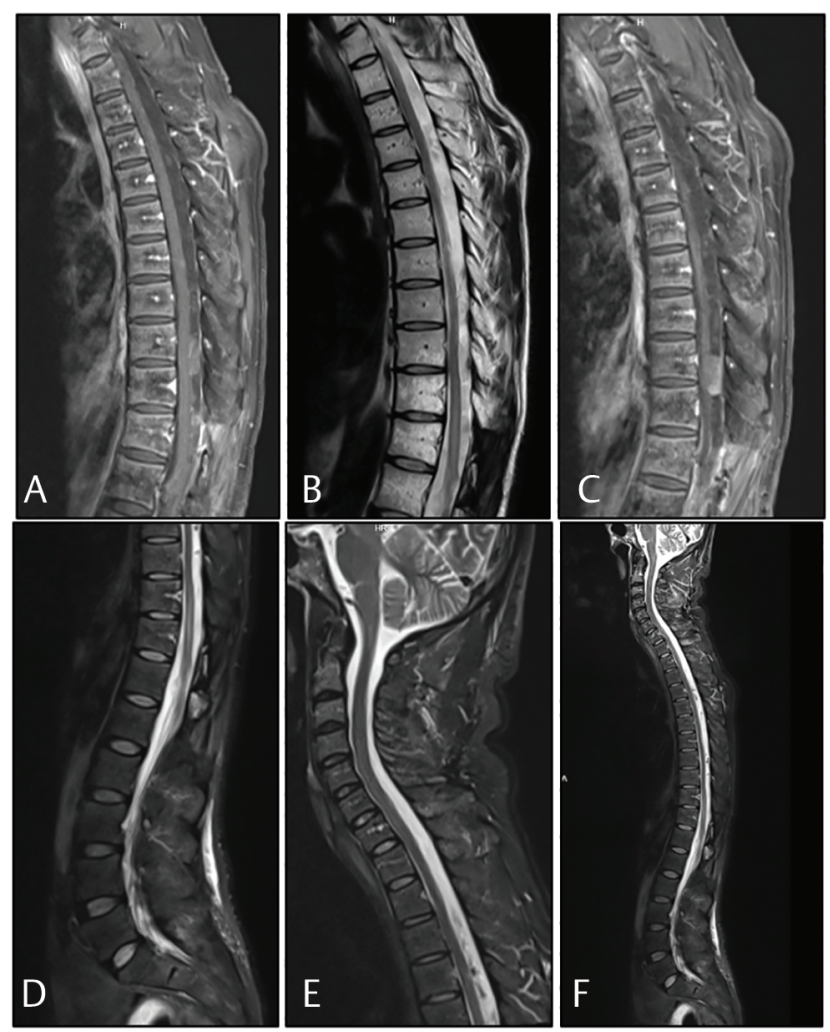

Fig. 2 (A-F) Five months after second radiotherapy combined with chemotherapy. Spinal MRI shows pronounced regression of hyperintense lesions in spinal meninges, especially in cervical level. Optimal spinal cord diameter has been established with decreased compression and narrowing.

It is postulated that CSF is involved in spread of tumor cells along CNS. ${ }^{9}$ In the present case, diffuse contrast enhancement along the meninges of spinal cord represents meningeal dissemination. Cranial MRI did not show any lesion. On the other hand, lesions along the spinal meninges caused significant compression of the spinal cord and narrowing of the spinal canal posteriorly.

Survival time of spinal glioblastoma is short, ranging from 9 to 15 months. ${ }^{9}$ The most important predictor of prognosis is histological grade of the tumor. ${ }^{1}$ Even though radical resection is crucial in optimal management of spinal glioblastoma, it is mostly unachievable due to tumoral invasion into adjacent tissue. ${ }^{2,9}$ In our case, gross total excision of the tumor was performed, but due to the infiltrative course of the tumor, dissemination was inevitable.

Radiotherapy is the preferred adjuvant therapy in primary spinal glioblastoma, which is shown to increase survival time ${ }^{1,3}$. However, metastasis despite radiotherapy is not uncommon, as in a case presented by Yang et al, where a patient was operated for spinal glioblastoma and received both chemotherapy and radiotherapy. Six months later, patient presented with multiple foci in spinal cord and brain. Chemotherapy is suggested for progression of the disease after radiotherapy. ${ }^{2}$ Even though utilization of temozolomide is proven to be effective in intracranial glioblastoma, efficacy is uncertain for spinal cord glioblastoma. ${ }^{3}$ In the literature, regression of dissemination of the primary spinal cord glioblastoma is not reported yet. In our case, 5 months after radiotherapy and chemotherapy with temozolomide, radiological examination revealed shrinkage of metastatic lesions, especially in cervical level. This may suggest that adjuvant therapy decreases tumor load significantly, despite the prognosis being poor.

\section{Conclusion}

Primary spinal glioblastoma is a rare entity that has poor outcome and high mortality. Currently, the treatment of these tumors consists of surgical resection, chemotherapy and radiotherapy, likewise intracranial glioblastoma. Meningeal dissemination of glioblastoma worsens the prognosis of patients. However, regression may be possible with radiotherapy and chemotherapy as shown in our case. Still, future studies are necessary to better understand the biology of spinal glioblastoma, and pathophysiology of its dissemination, in order to increase survival.

\section{Conflict of Interest \\ None declared.}

\section{References}

1 Randakevičienė G, Gleiznienė R, Basevičius A, Lukoševičius S. An extremely rare case of glioblastoma multiforme of the spinal cord. Medicina (Kaunas 2013;49(5):242-245

2 Chamberlain MC, Johnston SK. Recurrent spinal cord glioblastoma: salvage therapy with bevacizumab. J Neurooncol 2011;102(3):427-432

3 Cheng L, Yao Q Ma L, et al. Predictors of mortality in patients with primary spinal cord glioblastoma. Eur Spine J 2020;29(12):3203-3213

4 Noh JH, Lee MH, Kim WS, et al. Optimal treatment of leptomeningeal spread in glioblastoma: analysis of risk factors and outcome. Acta Neurochir (Wien 2015;157(4):569-576

5 Sibanda Z, Farahani N, Ogbonnaya E, Albanese E. Glioblastoma multiforme: a rare case of spinal drop metastasis. World Neurosurg 2020;144:24-27

6 Takara E, Ide M, Yamamoto M, Imanaga H, Jimbo M, Imai M. [Case of intracranial and spinal dissemination of primary spinal glioma]. No Shinkei Geka 1985;13(3):301-305. Japanese. PMID: 2989721

7 König SA, Roediger T, Spetzger U. Treatment of recurrent primary spinal glioblastoma multiforme-case report. J Neurol Surg A Cent Eur Neurosurg 2012;73(4):256-261

8 Schwaninger M, Patt S, Henningsen P, Schmidt D. Spinal canal metastases: a late complication of glioblastoma. J Neurooncol 1992;12(1):93-98

9 Ozgiray E, Akay A, Ertan Y, Cagli S, Oktar N, Ozdamar N. Primary glioblastoma of the medulla spinalis: a report of three cases and review of the literature. Turk Neurosurg 2013;23(6):828-834 